\title{
Fragmentation of Ions in a Low Pressure Linear Ion Trap
}

\author{
Bruce A. Collings \\ MDS SCIEX, Concord, Ontario, Canada
}

\begin{abstract}
The efficiency of in-trap fragmentation in a low-pressure linear ion trap (LIT), using dipolar excitation, is dependent upon the choice of both the excitation $q$ and the drive frequency of the quadrupole. In the work presented here, fragmentation efficiencies have been measured as a function of excitation $q$ for drive frequencies of $816 \mathrm{kHz}$ and $1.228 \mathrm{MHz}$. The experiments were carried out by fragmenting reserpine $(609.23 \rightarrow 448.20$ Th and $397.21 \rightarrow 365.19$ Th transitions) and caffeine $(195 \rightarrow 138$ Th and $138 \rightarrow 110$ Th transitions). The data showed that the onset of efficient fragmentation occurred at a lower Mathieu $q$ for the LIT operated at $1.228 \mathrm{MHz}$ when compared with the LIT operated at $816 \mathrm{kHz}$. A comparison of the fragmentation efficiency curves as a function of pseudo-potential well depth showed that the onset of fragmentation is independent of the drive frequency. In addition, a comparison of the fragmentation efficiency curves showed that all four of the precursor ions fragmented within a range of four $\mathrm{V}$ of pseudo-potential well depth. The choice of an appropriate excitation $q$ can then be determined based upon a minimum pseudo-potential well depth, quadrupole field radius, drive frequency, and the mass of interest. Fragmentation efficiencies were also found to be significantly greater when using the higher drive frequency. (J Am Soc Mass Spectrom 2007, 18, 1459-1466) (c) 2007 American Society for Mass Spectrometry
\end{abstract}

$\mathrm{T}$ The development of the low-pressure linear ion trap (LIT) [1] and mass selective axial ejection (MSAE) [2] enabled the development of the hybrid LIT triple quadrupole mass spectrometer. In this device, the second mass analyzing quadrupole doubles as the LIT. The LIT typically operates at pressures in the $3-5 e-5$ torr range, which is significantly lower than the mTorr pressure regime typically used for the operation of 3-D ion traps [3,4] and other high-pressure LITs $[5,6]$. This difference is a key factor in how in-trap fragmentation of ions, through the use of dipolar excitation, is performed [7]. The performance of 3-D ion traps in the mTorr regime have been well characterized and understood with regard to fragmentation efficiency, type of collision gas, excitation $q$, and a variety of models [8-13].

In the low-pressure LIT the collision frequency is low enough, about tens to a few $100 \mu$ s between collisions, that the ion experiences several rf cycles between collision events with the background gas. It becomes necessary to take advantage of the presence of higher order fields, occurring naturally with the round rod electrodes used in the construction of the quadrupole, to keep the ions confined radially within the LIT during the fragmentation process. This also necessitates the use of low excitation amplitudes, about several tens of $\mathrm{mV}$ amplitudes, and excitation periods about a few tens to a couple of hundred ms. The duration of the excitation

Address reprint requests to Dr. B. A. Collings, MDS SCIEX, 71 Four Valley Dr., Concord, Ontario L4K 4V8, Canada. E-mail: Bruce.Collings@sciex.com periods can be reduced and the excitation amplitudes increased without loss of the precursor ion by increasing the content of the higher order fields. This can be done with the use of additional electrodes along the length of the LIT [14] or by distorting the physical dimensions of the quadrupole electrodes for the purposes of introducing additional higher order fields into the radial trapping potential $[4,8,15]$.

It was noted by Michaud et al. [15] that in their experiments the fragmentation efficiency of reserpine at $q=0.20$ was significantly less efficient than that observed by Collings et al. [7] at a slightly higher excitation $q$ of 0.21 when using an LIT constructed from a conventional round rod quadrupole array. It was speculated that one contributing factor may have been the difference in drive frequencies, $768 \mathrm{kHz}$ for their experiments and $816 \mathrm{kHz}$ for the experiments of Collings et al., leading to a significant difference in the pseudopotential well depths, 6.4 and $8.0 \mathrm{eV}$ respectively. This corresponds to an increase of $25 \%$ in the pseudopotential well depth which helps to retain the precursor and fragment ions within the LIT leading to higher fragmentation efficiencies.

This paper will detail experimental measurements on the efficiency of fragmentation as a function of the excitation $q$ and the quadrupole drive frequency for precursor and fragment ions of caffeine and reserpine. The data will show that fragmentation efficiency curves expressed as a function of the pseudo-potential well depth, instead of the excitation $q$, are independent of the quadrupole drive frequency. 


\section{Experimental}

Experiments were carried out on two separate hybrid triple quadrupole linear ion trap mass spectrometers. The first was a modified 4000 QTRAP (AB/MDS SCIEX, Concord, Ontario, Canada) described elsewhere [7, 14]. Briefly, ions were formed using an electrospray ionization source. The ions were transported through an aperture in a curtain plate, then a sampling orifice, a skimmer and into an rf-only quadrupole (Q0) operated at 7 mTorr of nitrogen. Nitrogen was used as a curtain gas. Ions exiting the Q0 quadrupole passed through a differential pumping aperture and into the first mass analyzing quadrupole (Q1). Ions were mass selected in $\mathrm{Q} 1$, accelerated to ion energies between 10 and $43 \mathrm{eV}$ into a nitrogen filled collision cell (Q2) operated at a pressure of $\sim 5 \mathrm{mTorr}$. The parent and fragment ions next entered a mass analyzing quadrupole (Q3) which also performed the function of a linear ion trap (LIT). At the end of Q3 was a gridded exit lens followed by an ion detector. The experiments discussed in this paper used Q1 in mass analyzing mode and Q3 exclusively as a LIT. In trap mode the pressure in the LIT was maintained at 3.3e- 5 torr of nitrogen derived from both the curtain gas and primarily the Q2 collision cell. Ions were trapped in the LIT by raising the DC potentials on the exit lens and a set of short rf-only quadrupoles located between the Q2 collision cell and the Q3 quadrupole. Ions were scanned out of the LIT at a rate of $1000 \mathrm{Da} / \mathrm{s}$ using the technique of MSAE [2].

The second hybrid triple quadrupole LIT mass spectrometer was a research test bed that employed a LIT identical to that in the 4000 QTRAP described above. The important difference, for the purposes of this study, was the quadrupole drive frequency. The 4000 QTRAP operated at a drive frequency of $816 \mathrm{kHz}$ while this instrument operated at $1.228 \mathrm{MHz}$. The LIT in the latter instrument was also operated at a pressure of $3.6 \mathrm{e}-5$ torr of nitrogen. The difference in pressure between the two instruments was determined from a post experiment calibration of the vacuum gauges against a secondary standard. This difference is expected to have a minor affect on the experimental results. Since one aspect of the experiments was to demonstrate the effect of drive frequency on fragmentation efficiency it was important to maintain similar ion densities within the LIT when comparing data collected on the two instruments. This was accomplished by adjusting the LIT fill times and detuning of selected ion optics to obtain similar ion signals.

In-trap fragmentation of ions was carried out using the technique of dipolar excitation [7]. This was accomplished using the $\mathrm{MS}^{3}$ scan function of the QTRAP instruments. In this function the precursor ion is mass selected in Q1 and sent into the Q2 collision cell at a desired collision energy. The precursor ion and the resulting fragment ions were collected in the LIT. An ion of interest was then selected in the LIT by applying the Q3 resolving DC for a short period of time (1 ms). The drive rf amplitude on Q3 was then lowered to a value that brought the ion of interest to a desired $q$ value for excitation. The ion of interest was fragmented, allowed to cool for a period of $100 \mathrm{~ms}$ and the spectrum recorded. The $100 \mathrm{~ms}$ cooling period was sufficient to ensure that fragmentation, due to a unimolecular dissociation process, was complete. Excitation periods were varied from 50 to $200 \mathrm{~ms}$ with the majority of the experiments using a period of $100 \mathrm{~ms}$. Excitation amplitudes were varied up to $60 \mathrm{mV}$ (zero to peak, pole to pole) and ions were excited at Mathieu $q$ values ranging from 0.1 to 0.5 . All excitation amplitudes reported in the paper were measured zero to peak, pole to pole.

Solutions of caffeine (100 pg/ $\mu \mathrm{l}, 50 / 50$ methanol/ water plus $0.1 \%$ formic acid) and reserpine $(10 \mathrm{pg} / \mu \mathrm{l}$ and $100 \mathrm{pg} / \mu \mathrm{l}, 50 / 50$ water/acetonitrile with $0.1 \%$ acetic acid) were used in these experiments. The solutions were infused at a rate of $7 \mu \mathrm{l} / \mathrm{min}$ and the ions were created using electrospray ionization. In-trap fragmentation experiments were carried out on the ions reserpine $(609.23 \rightarrow 448.20 \mathrm{Th}$ and $397.21 \rightarrow 365.19 \mathrm{Th}$ transitions) and caffeine $(195 \rightarrow 138 \mathrm{Th}$ and $138 \rightarrow 110 \mathrm{Th}$ transitions). Fragmentation efficiencies were calculated using the intensity of the major fragment and precursor ions. This method of comparison was used instead of integrating the sum of all the fragment ions because of the effects of the low mass cut-off when the excitation $q$ was increased. As the excitation $q$ increases the low mass cut-off also increases resulting in the loss of the low mass fragments through radial instability within the LIT. Choosing a fragment that was always above the low mass cut-off allowed for a comparison of fragmentation efficiencies over a range of excitation $q$ 's.

\section{Ion Trajectory Simulations}

It was necessary to know the secular frequency of an ion trapped within the LIT constructed of round rods to enable relatively easy location of the excitation resonance. Equations for calculating the frequencies of ion motion are available for ions trapped within pure quadrupolar fields but are not readily available when higher order fields are present. An ion trajectory simulator was used to determine the difference in secular frequencies between a quadrupole constructed of round rods and one constructed of hyperbolic rods. The ion trajectory simulator has been previously described in references [2, 7].

All experimental data were collected using quadrupoles constructed of round rods. The potential fields created by the round rods contain higher order multipole terms [16], described by eq 1 , which causes a shift in the ion's secular frequency compared to that of a quadrupole constructed from hyperbolic shaped rods. A general expression for a two-dimensional multipole field can be written as 


$$
\Phi(x, y)=\sum_{n=0}^{\infty} \phi_{n}(x, y)=\sum_{n=0}^{\infty} A_{n} \operatorname{Re}\left(\frac{x+i y}{r_{0}}\right)^{n}
$$

where $2 \mathrm{n}$ is the number of poles in a particular term and, for example, $A_{2}$ is the amplitude of the quadrupole contribution. In both the experiments and simulations, the ratio of the rod radius to the field radius was 1.126 . This ratio results in coefficients of $A_{2}=1.001462, A_{6}=$ $0.001292, A_{10}=-0.002431$, and $A_{14}=-0.0002975$ with all other terms for which $n<14$ equal to zero. The magnitude of the coefficients for $n>2$ are enough to cause significant shifting of the ion's secular frequency from the case where only $A_{2}$ is non-zero (a pure quadrupolar field). An ion trajectory simulator, described in reference [7], was used to determine the resonance frequency of ions confined within a quadrupole modeled using round rods and a quadrupole modeled using hyperbolic shaped electrodes. The simulations were carried out over a range of 0.15 to 0.85 in $q$ space. This covers the range of $q$ normally used for ion excitation leading to both fragmentation and the ejection of ions using MSAE, which is normally operated at $q \approx 0.85$. The secular frequency of an ion at a particular $q$ was determined by summing up the total energy loss of an ion during the excitation process [7]. ÅThe Atotal energy loss is simply the amount of kinetic energy that the ion loses in inelastic collisions with the background gas. Inelastic collisions with nitrogen were simulated using the hard sphere model. The simulations used mass 609 , a collision cross-section of $280 \AA^{2}$, an excitation period of typically $10 \mathrm{~ms}$, excitation amplitudes ranging from 10 to $40 \mathrm{mV}$, a pressure of $3.5 \mathrm{e}-5$ torr of mass 28 and a total of 10 trajectories at each excitation frequency. The starting conditions of the ions were defined by first randomly confining 10 ions to within a $1 \mathrm{~mm}$ radius of the quad axis with thermal kinetic energy. The ions were then cooled for a period of $5 \mathrm{~ms}$ at 5 mTorr of nitrogen. The final conditions of these ions were then used as input into the simulator. The resonant frequency of the ion was then found by fitting a plot of the total energy loss versus excitation frequency

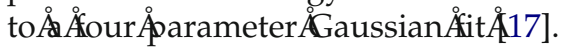

\section{Results and Discussion}

\section{Simulation Results}

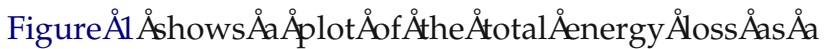
function of excitation frequency for the excitation of 609.23 Th at $q=0.23685$ in a round rod quadrupole operating at $816 \mathrm{kHz}$ using an excitation amplitude of $20 \mathrm{mV}$ and an excitation period of $10 \mathrm{~ms}$. The total energy loss is at a maximum when the ion is in resonance with the excitation signal. This occurs at 69

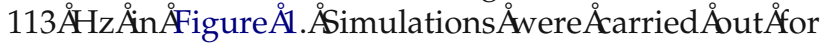
several q values over the range 0.15022 to 0.85124 . In all cases the excitation amplitude and excitation period were held constant which led to frequency response profiles of constant width. The resonant frequencies

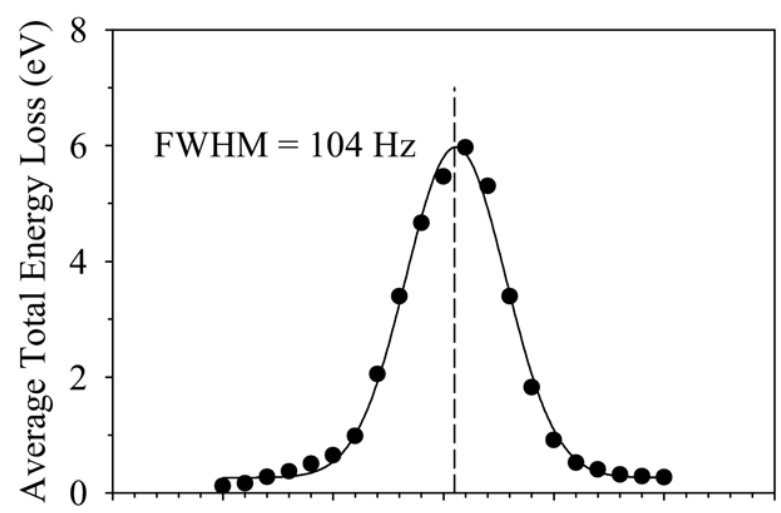

68800689006900069100692006930069400

\section{Excitation Frequency $(\mathrm{Hz})$}

Figure 1. Average total energy loss for the excitation of $609.23 \mathrm{Th}$ at $q=0.2365$ and $\Omega=816 \mathrm{kHz}$. The broken vertical line represents the center of the resonance as determined by a four parameter Gaussian fit. An excitation amplitude and period of $20 \mathrm{mV}$ and 10 $\mathrm{ms}$, respectively, were used for the simulation.

were then compared to the secular frequencies of ions confined in a purely quadrupolar field which were calculated analytically. As a check on the accuracy of the simulator, simulations were carried out for ions confined in a quadrupole constructed of hyperbolic shaped electrodes at $q=0.23685$ and $q=0.70703$. The resonant frequencies determined through simulation matched the calculated secular frequencies to within 2 $\mathrm{Hz}$, corresponding to less than $2 \%$ of the typical simulation FWHM.

Calculation of the secular frequency shift at a different drive frequency is simply a matter of recognizing that the shift is proportional to the secular frequency of the ion in a pure quadrupolar field. This was shown by

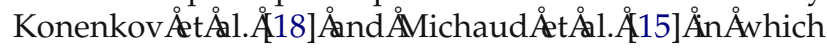
frequency shifts were calculated for added hexapole and octopole terms to the field, respectively. The secular frequency of an ion confined in a quadrupolar field (a hyperbolic rod quadrupole) can be found analytically using

$$
\omega_{0}=\beta \frac{\Omega}{2}
$$

where $\Omega$ is the angular frequency of the RF drive and $\beta$ is a function of the Mathieu stability parameters $a$ and $q$. The parameter $\beta$ is calculated using the continued

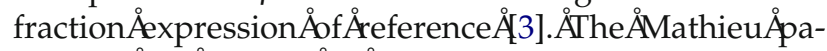

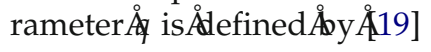

$$
q=\frac{4 e V_{r f}}{m r_{0}^{2} \Omega^{2}}
$$

where $e$ is the electronic charge, $V_{r f}$ is the $\mathrm{rf}$ amplitude measured pole to ground, $m$ is the mass of the ion and $r_{0}$ is the field radius of the quadrupole. The existence of 
a non-zero $A_{2}(=1.001462)$ multipole coefficient means that the field acts as if $V_{r f}$ is 1.001462 times greater than in a pure quadrupolar field. Including this factor in the expression for $q$ leads to the expression

$$
q=\frac{4 e A_{2} V_{r f}}{m r_{0}^{2} \Omega^{2}} .
$$

To determine an ion's secular frequency shift, caused by the introduction of higher order fields, the ion's secular frequency in a pure quadrupolar field should be determined using the value of $q$ obtained from eq 4 . Using $q$ calculated from eq 3 leads to secular frequencies that are significantly lower, thus giving seemingly larger secular frequency shifts. In fact, this led to an initial misinterpretation of the data when the experimental results were compared to the calculated secular frequencies. The Mathieu parameter $a$ was equal to zero for all the experiments discussed in this paper.

Figure $\AA 2 \AA$ shows $\AA$ A ${ }_{\text {plot }}$ Af $\AA$ the $A$ difference $\AA$ in $\AA$ secular frequency between ions confined in a round rod quadrupole and a hyperbolic rod quadrupole as a function of $q$ when using a drive frequency equal to $816 \mathrm{kHz}$. The data were collected with excitation amplitudes ranging from 10 to $40 \mathrm{mV}$ while keeping the excitation period constant at $10 \mathrm{~ms}$. The $20 \mathrm{mV}$ data shows that the secular frequency difference increases with $q$ to $66 \mathrm{~Hz}$ at $q=0.85124$ where the secular frequency is $316439 \mathrm{~Hz}$ for ions in the round rod quadrupole and $316373 \mathrm{~Hz}$ for ions in the hyperbolic rod quadrupole. At $q=0.23685$ the frequency difference is only $10 \mathrm{~Hz}$ which is well within the FWHM of $104 \mathrm{~Hz}$ for the frequency response profile ̊hown Ån Åigure $\AA$. Åhe Åxcitation Åmplitude varied for simulations at $q=0.23685$ and $q=0.85124$. In

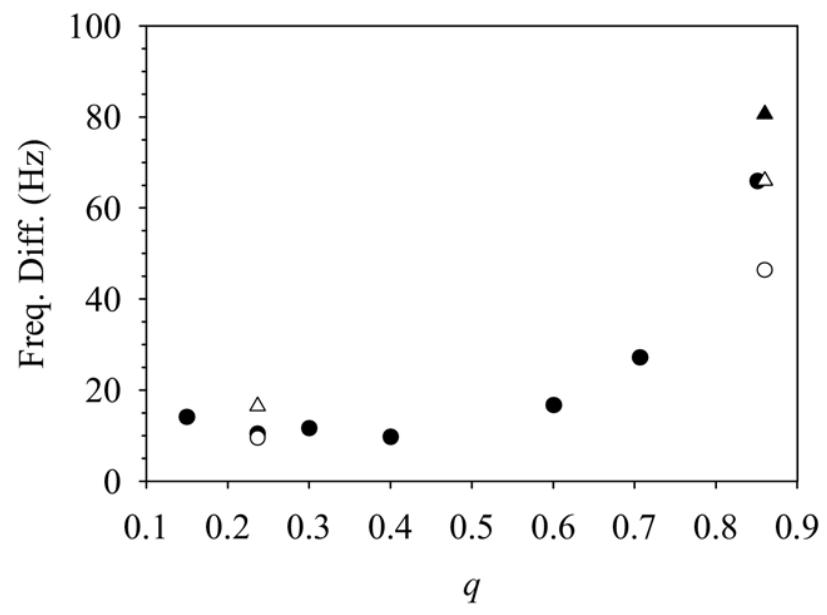

Figure 2. A plot of the secular frequency difference between an ion confined to a quadrupole constructed of hyperbolic shaped rods and one constructed of round rods as a function of $q$. The secular frequencies are higher for ions confined in the round rod quadrupole. Data are shown for excitation amplitudes of $10 \mathrm{mV}$ (open circles), $20 \mathrm{mV}$ (filled circles), $30 \mathrm{mV}$ (open triangles) and 40 $\mathrm{mV}$ (filled triangle). The data were obtained using a drive frequency of $816 \mathrm{kHz}$.
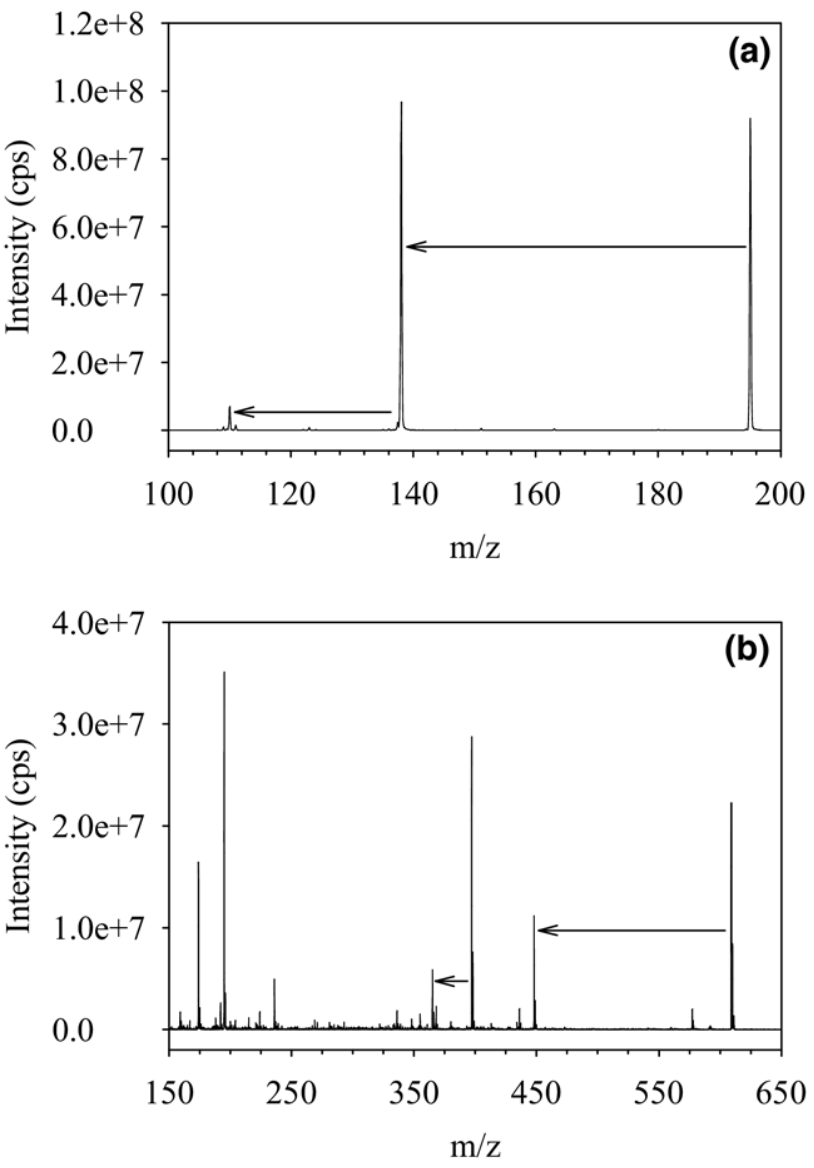

Figure 3. Enhanced product ion mass spectra for reserpine (609.23 Th) at a collision energy of $40 \mathrm{eV}$ and for caffeine (195 Th) at $25 \mathrm{eV}$.

all cases the ion trajectories completed the $10 \mathrm{~ms}$ time limit without terminating on a quadrupole rod. The data shows that the ion's secular frequency shifts upward with increasing excitation amplitude. In the cases that were simulated, the degree of shifting was less than the typical FWHM of the experimental frequency response profile. In general, excitation in a low-pressure LIT requires excitation amplitudes which are typically in the range of 10 to $100 \mathrm{mV}$. Calculation of the resonance frequency based upon the $q$ value determined from eq 4 gives a lower limit to the resonant frequency that will also most likely be within the FWHM of the frequency response profile for a typical ion. This knowledge provides for the ability to change the excitation $q$ and find the experimental resonance with minimal error and time.

\section{Experimental Results}

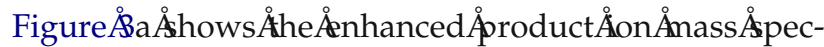
trum of caffeine, $195 \mathrm{Th}$, at a collision energy (CE) of 25

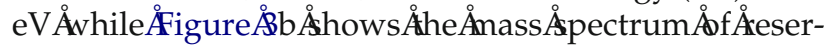
pine, 609.23 Th, at $\mathrm{CE}=40 \mathrm{eV}$. The arrows show the transitions that were monitored in each of the experiments. In an enhanced product ion mass spectrum a 
precursor mass is selected in the Q1 quadrupole, accelerated into the Q2 collision cell to cause fragmentation and the fragments are collected in the Q3 LIT for mass

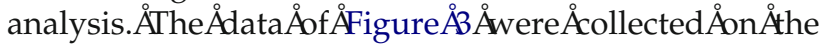
$1.228 \mathrm{MHz}$ instrument and are a sum of 50 scans. The caffeine spectrum has a major fragment at 138 Th that was used for in-trap fragmentation in addition to intrap fragmentation of the 195 Th precursor ion. In the case of reserpine both the 609.23 Th precursor and the 397.21 Th fragment ion were selected for in-trap fragmentation.

Determination of the fragmentation efficiency, as a function of $q$, of an ion required a number of steps. The first step, after the ion of interest was created, was the determination of the location of the resonance for the chosen excitation $q$. This was accomplished by first calculating the location of the resonance in frequency space and then converting this value to an rf amplitude to be applied to the Q3 quadrupole. The required rf amplitude was then converted into a useful control parameter for the mass spectrometer, in this case a Q3 mass value which was called the Q3 set mass. The Q3 set mass was varied while monitoring the intensity of the peak of interest with the dipole excitation applied. The resonance was located by taking the point of maximum depletion. Typically the resonance was mapped using an excitation amplitude that would result in $50 \%$ to $80 \%$ depletion of the ion of interest.

To obtain the fragmentation efficiency it is necessary to measure the intensity of the precursor and fragment ions as a function of excitation amplitude. This is shown

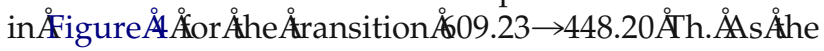
excitation amplitude was increased the intensity of the precursor ion decreased and the intensity of the fragment increased to a maximum. At too high an amplitude ejection of the ions from the LIT occurred and resulted in a decrease in both the precursor and fragment ion intensities. For the purpose of this study the fragmentation efficiency was calculated using the frag-

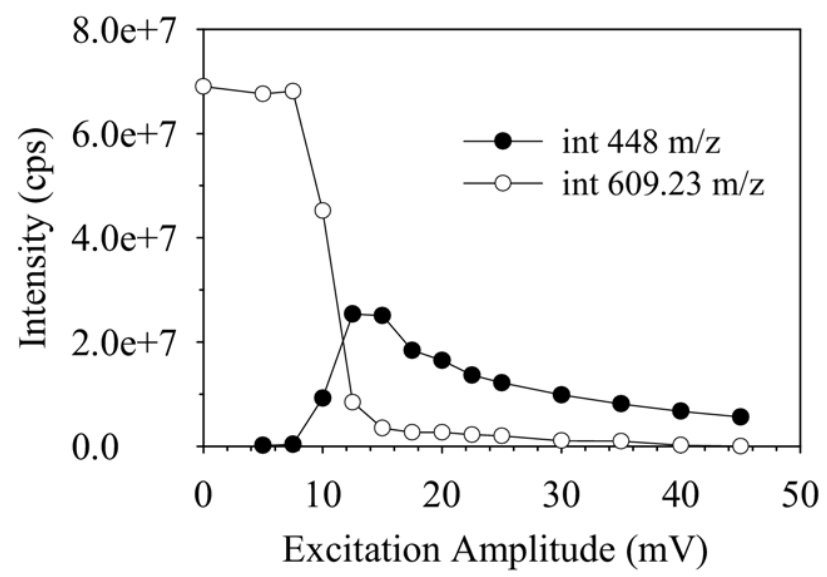

Figure 4. Reserpine precursor ion (609.23 Th) and fragment ion $(448.20 \mathrm{Th})$ intensity as a function of excitation amplitude at $q=$ 0.304 . The excitation period was $100 \mathrm{~ms}$, the pressure was $3.6 \mathrm{e}-5$ torr and the drive frequency $1.228 \mathrm{MHz}$.

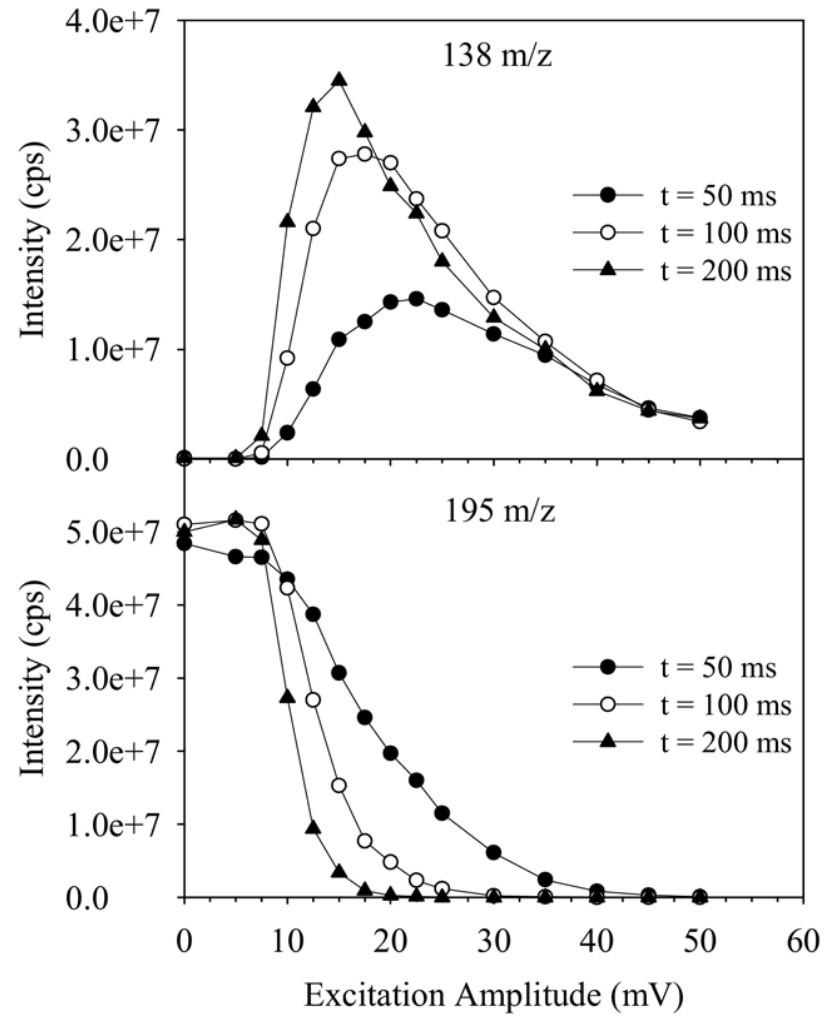

Figure 5. Caffeine precursor ion (195 Th) and fragment ion (138 Th) intensity as a function of excitation amplitude at $q=0.205$. The pressure was $3.6 \mathrm{e}-5$ torr and the drive frequency $1.228 \mathrm{MHz}$. The duration of the excitation period was varied from 50 to 100 to $200 \mathrm{~ms}$.

ment ion intensity at the maximum of the curve in

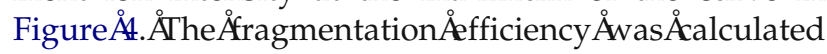
using

$$
\% \text { Frag eff }=\frac{\text { Maximum intensity of fragment }}{\text { Intensity of precursor }\left(V_{e x c}=0\right)} * 100 \%
$$

This procedure was performed for each percent fragmentation efficiency data point collected.

\section{Fragmentation Efficiency versus Excitation Period}

The choice of excitation period also affects the degree of fragmentation. Exciting for a longer period of time using low excitation amplitudes can lead to higher fragmentation efficiencies than when exciting at short periods using high excitation amplitudes. This is due to the onset of ejection of the ions at the higher excitation

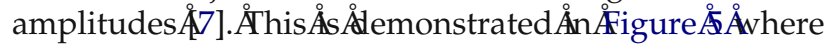
caffeine (195 Th) was fragmented at $q=0.205$ while monitoring the intensity of the 138 Th fragment. The maximum fragmentation efficiency occurred using an excitation period of $200 \mathrm{~ms}$ and an amplitude of $15 \mathrm{mV}$. Decreasing the excitation period to $50 \mathrm{~ms}$ results in the maximum occurring at an amplitude of $22.5 \mathrm{mV}$, how- 


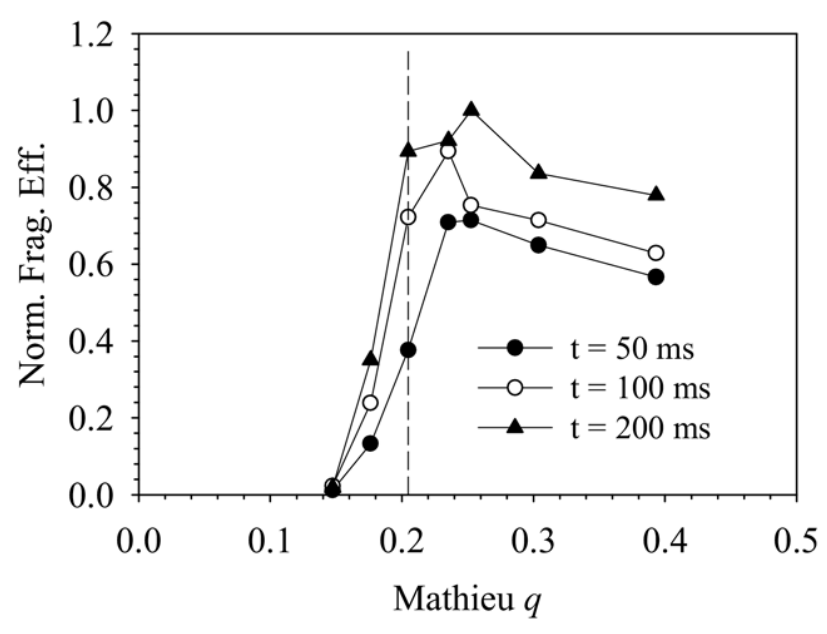

Figure 6. Normalized fragmentation efficiency as a function of the excitation $q$ and the excitation period for transition $195 \rightarrow 138$ Th. The data are normalized to the maximum of the $t=200 \mathrm{~ms}$ data. The broken vertical line indicates the $q$ value and the data that Åvas

ever, the maximum intensity of the fragment ion has decreased by more than a factor of two. For the purposes of collecting fragmentation efficiencies as a function of excitation $q$ an excitation period of $100 \mathrm{~ms}$ was chosen. This is a compromise between duty cycle and fragmentation efficiency.

The choice of excitation period also has an effect upon the shape of the fragmentation efficiency as a

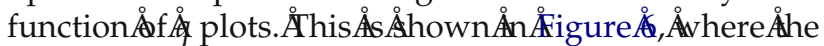
normalized fragmentation efficiency is plotted for the $195 \rightarrow 138$ Th transition of caffeine. The data has been normalized to the maximum of the $t=200 \mathrm{~ms}$ data. The vertical broken line indicates the excitation $q$ value and

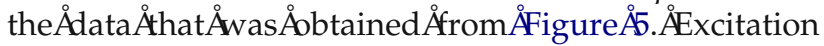
using a longer period of time allows the use of lower excitation amplitudes which in turn reduces the degree of ejection. This causes the fragmentation efficiency curve to be shifted slightly towards lower excitation $q$ values. The fragmentation efficiency increases to a maximum when the amount of ion ejection is reduced due to the increase in the pseudo-potential well depth. The pseudo-potential well depth is directly related to both the confinement $q$ and the rf amplitude by the expression 120$]$

$$
\bar{D}=\frac{q V_{r f}}{4}
$$

where $V_{r f}$ is obtained by rearranging eq 4 to obtain

$$
V_{r f}=\frac{q m r_{0}^{2} \Omega^{2}}{4 e A_{2}} .
$$

Increasing the drive frequency requires that $V_{r f}$ is increased to maintain the same excitation $q$. This results in an increased pseudo-potential well depth which translates into better ion confinement.

\section{Fragmentation Efficiency versus Drive Frequency}

Figure Ka Åhows Åragmentation excitation of caffeine (195 Th to $138 \mathrm{Th}$ ) as a function of $q$ and drive frequency. The data has been normalized to unity at the maximum of each curve with a maximum fragmentation efficiency of $75 \%$ for the $1.228 \mathrm{MHz}$ drive frequency data and $53 \%$ for the $816 \mathrm{kHz}$ drive frequency data. The efficiency curve shifts towards lower $q$ when the drive frequency is increased from $816 \mathrm{kHz}$ to $1.228 \mathrm{MHz}$. When the data are plotted as a function of D the Áwo đurves

The maximum fragmentation efficiency was found to be greater at the higher drive frequency for all of the fragmentation channels that were monitored. This effect

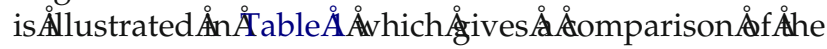
maximum fragmentation efficiency as a function drive frequency. A number of parameters change with an increase in drive frequency. Among them an increase in secular frequency for a particular excitation $q$, which is expected based upon an examination of eq 2, and an increase in the acceptance of the quadrupole.
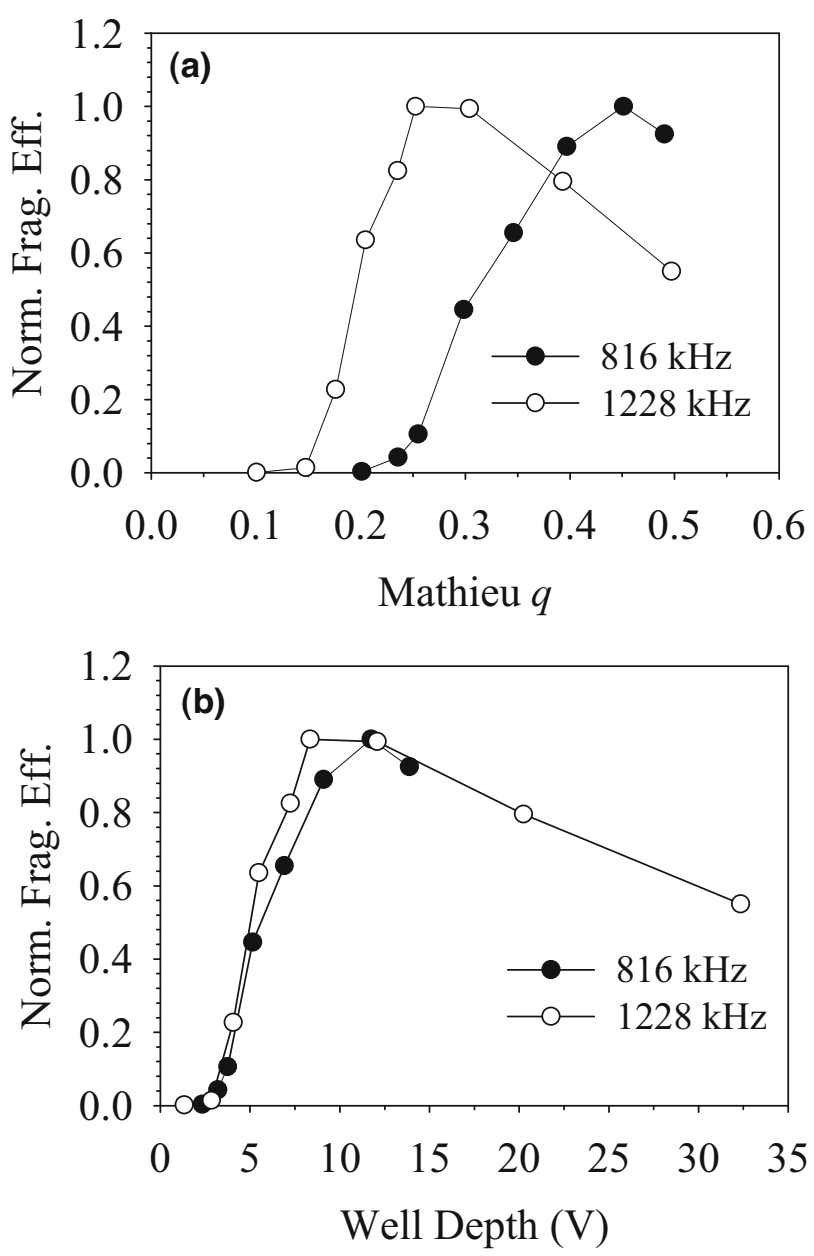

Figure 7. (a) Normalized fragmentation efficiency of Caffeine as a function of $q$ (for the $195 \rightarrow 138$ Th transition) and drive frequency. (b) The normalized fragmentation efficiency data of (a) replotted as a function of pseudo-potential well depth. 
Table 1. Maximum fragmentation efficiencies as a function of drive frequency for the transitions monitored

\begin{tabular}{lcc}
\hline $\begin{array}{c}\text { Transition } \\
\text { monitored }\end{array}$ & $\begin{array}{c}\Omega=816 \mathrm{kHz} \\
\% \text { frag eff }\end{array}$ & $\begin{array}{c}\Omega=1.228 \mathrm{MHz} \\
\% \text { frag eff }\end{array}$ \\
\hline \hline $138 \rightarrow 110$ & $12.0 \%$ & $26.7 \%$ \\
$195 \rightarrow 138$ & $52.5 \%$ & $75.0 \%$ \\
$397.21 \rightarrow 365.19$ & $19.6 \%$ & $24.6 \%$ \\
$609.23 \rightarrow 448.20$ & $33.0 \%$ & $44.4 \%$ \\
\hline
\end{tabular}

The secular frequency is dependent upon the parameter $\beta$ which is a function of $q$. Since $\bar{D}$ is a function of $q$ it can be realized that $\beta$ is also a function of $\bar{D}$ implying that the difference in secular frequency can be removed by plotting the fragmentation efficiency data as a function $\AA$ f

Increasing the drive frequency will lead to an increase in the acceptance of the quadrupole. The acceptance of the quadrupole is usually expressed in terms of the reduced variables $u$ ( $x$ or $y$ expressed in terms of the field radius and the velocity) and $\dot{u}$ measured in units of field radii per RF cycle, i.e., in units of $r_{0} f$ [20].ÅThe acceptance of the $x$ and $y$ coordinates each scale as $r_{0}^{2} f$ giving a combined acceptance for $x$ and $y$ of $r_{0}^{4} f^{2}$. An increase in drive frequency from $816 \mathrm{kHz}$ to $1.228 \mathrm{MHz}$ gives an increase in acceptance of $\times 2.26$. The increased acceptance would contribute to the ability of the LIT to retain ions that are scattered or fragmented into regions of phase space that are outside of the acceptance of the $816 \mathrm{kHz}$ drive frequency leading to an increase in fragmentation efficiency.

\section{Effect of Mass}

Figure caffeine $(195 \rightarrow 138$ Th and $138 \rightarrow 110$ Th transitions) and reserpine $(609.23 \rightarrow 448.20 \mathrm{Th}$ and $397.21 \rightarrow 365.19 \mathrm{Th}$ transitions) at $816 \mathrm{kHz}$ plotted as a function of excitation $\AA$, distinction between the onset of fragmentation for the reserpine related ions and the caffeine related ions in $q$ space. However, there is no real distinction between the curves for reserpine and its fragment in $q$ space, nor for caffeine and its fragment. When the data are plotted as a function of $\bar{D}$ the curves overlap to within a range of about $4 \mathrm{~V}$ with no clear mass trend with the exception of the lower $\overline{\mathrm{D}}$ thresholds for the lower $\mathrm{m} / \mathrm{z}$ precursors of reserpine and caffeine. However, with the limited dataset could be a useful starting point when determining a minimum excitation $q$ value for efficient fragmentation. The choice of $\overline{\mathrm{D}}$ is based on the point in the plots where the fragmentation efficiency rises to a maximum for all masses. Combining eq 6 and eq 7 gives the following expression for a minimum excitation $q$ for efficient fragmentation.

$$
q=\frac{16 e A_{2} \bar{D}}{m r_{0}^{2} \Omega^{2}}
$$

The onset of fragmentation is complicated by a number of factors including the rate of fragmentation and the minimum pseudo-potential well depth required to keep the ion confined during the excitation process. (It should he Ånoted Åhat Åhe Aata Aor Figure Avas Alerived using excitation amplitudes that varied from 20 to 45 $\mathrm{mV}$, which were the excitation amplitudes required to obtain the maximum fragmentation efficiencies.) The rate of fragmentation is governed by several factors ranging from the type of bond that is broken, the number of degrees of freedom available to the ion for storage of internal energy, the excitation period and the rate of internal energy uptake to the ion through collisions with the collision gas. The rate of internal energy uptake is also affected by the excitation amplitude, the excitation frequency, the gas density and the masses Âthe tion will be necessary before a more complete under-
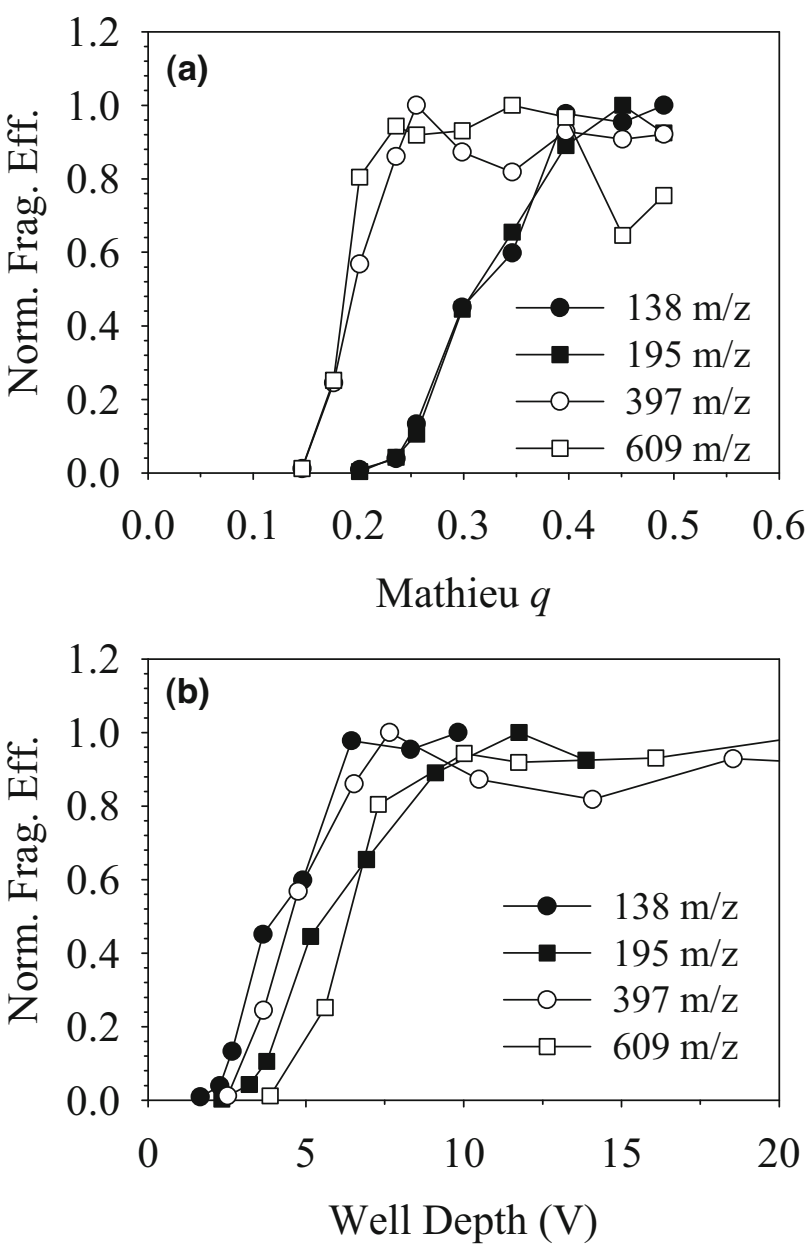

Figure 8. (a) Normalized fragmentation efficiency curves as a function of $q$ and (b) pseudo-potential well depth for different masses. The data were collected using a drive frequency of 816 $\mathrm{kHz}$. 
standing of all the factors affecting the onsets of fragmentation can be given, which is beyond the intended scope of this paper.

\section{Conclusions}

It has been shown that fragmentation efficiencies of trapped ions, using dipolar excitation, will have a Mathieu $q$ value dependence that is drive frequencydependent. This dependency is removed when the data are plotted as a function of the pseudo-potential well depth. It has also been shown that the fragmentation efficiency curves, for a number of different masses, show different onsets of fragmentation when plotted as a function of $q$ at a single drive frequency. Plotting the data as a function of the pseudo-potential well depth shows a spread of a few $\mathrm{eV}$ for the onset of fragmentation. This suggests that the pseudo-potential well depth may be used to select a minimum $q$ value for efficient fragmentation based upon the quadrupole field radius, drive frequency, and the mass of interest.

\section{Acknowledgments}

The author acknowledges the comments made by Don Douglas a few years ago, which prompted this study. The author also thanks Jim Hager and Yves LeBlanc for reviewing and criticizing this manuscript before submission.

\section{References}

1. Hager, J. W. A New Linear Ion Trap Mass Spectrometer. Rapid Commun. Mass Spectrom. 2002, 16, 512-526.
2. Londry, F. A.; Hager, J. W. Mass Selective Axial Ion Ejection from a Linear Quadrupole Ion Trap. J. Am. Soc. Mass Spectrom. 2003, 14 1130-1147.

3. March, R. E. An Introduction to Quadrupole Ion Trap Mass Spectrometry. J. Mass Spectrom. 1997, 32, 351-369.

4. March, R. E. Quadrupole Ion Trap Mass Spectrometry: Theory, Simulation, Recent Developments and Applications. Rapid Commun. Mass Spectrom. 1998, 12, 1543-1554.

5. Schwartz, J. C.; Senko, M. W.; Syka, J. E. P. A Two-Dimensional Ion Trap Mass Spectrometer. J. Am. Soc. Mass Spectrom. 2002, 13, 659-669.

6. Hashimoto, Y.; Hasegawa, H.; Baba, T.; Waki, I. Mass Selective Ejection by Axial Resonant Excitation from a Linear Ion Trap. J. Am. Soc. Mass Spectrom. 2006, 17, 685-690.

7. Collings, B. A.; Stott, W. R.; Londry, F. A. Resonant Excitation in a Low-Pressure Linear Ion Trap. J. Am. Soc. Mass Spectrom. 2003, 14 622-634.

8. Charles, M. J.; McLuckey, S. A.; Glish, G. L. Competition between Resonance Ejection and Ion Dissociation during Resonant Excitation in a Quadrupole Ion Trap. J. Am. Soc. Mass Spectrom. 1994, 5, 1031-1041.

9. Goeringer, D. E.; Asano, K. G.; McLuckey, S. A. Ion Internal Temperature and Ion Trap Collisional Activation: Protonated Leucine Enkephalin. Int. J. Mass Spectrom. 1999, 182/183, 275-288.

10. Goeringer, D. E.; McLuckey, S. A. Relaxation of Internally Excited High-Mass Ions Simulated Under Typical Quadrupole Ion Trap Storage Conditions. Int. J. Mass Spectrom. 1998, 177, 163-174.

11. Goeringer, D. E.; McLuckey, S. A. Kinetics of Collision-Induced Dissociation in the Paul Trap: A First-Order Model. Rapid Commun. Mass Spectrom. 1996, 10, 328-334.

12. Goeringer, D. E.; McLuckey, S. A. Evolution of Ion Internal Energy During Collision Excitation in the Paul Ion Trap: A Stochastic Approach. J. Chem. Phys. 1996, 104(6), 2214-2221.

13. Danell, R. M.; Danell, A. S.; Glish, G. L.; Vachet, R. W. The Use of Static Pressures of Heavy Gases Within a Quadrupole Ion Trap. J. Am. Soc. Mass Spectrom. 2003, 14, 1099-1109.

14. Collings, B. A. Increased Fragmentation Efficiency of Ions in a Low Pressure Linear Ion Trap with an Added dc Octopole. J. Am. Soc. Mass Spectrom. 2005, 16, 1342-1352.

15. Michaud, A.; Frank, A.; Ding, C.; Zhao, X.; Douglas, D. J. Ion Excitation in a Linear Quadrupole Ion Trap with an Added Octopole Field. J. Am. Soc. Mass Spectrom. 2005, 16, 835-849.

16. Douglas, D. J.; Glebova, T. A.; Konenkov, N. V.; Sudakov, M. Y. Spatial Harmonics of the Field in a Quadrupole Mass Filter with Circular Electrodes. Tech. Phys. 1999, 44, 1215-1219.

17. SigmaPlot ${ }^{\circledR}$ version 10.0; Systat Software Inc.: Point Richmond, CA.

18. Konenkov, N.; Londry, F.; Ding, C.; Douglas, D. J. Linear Quadrupoles with Added Hexapole Fields. J. Am. Soc. Mass Spectrom. 2006, 17, 1063-1073.

19. Dawson, P. H. Quadrupole Mass Spectrometry and Its Applications; AIP Press: Woodbury, NY, 1995; pp 9-64

20. Dehmelt, H. G. Radiofrequency Spectroscopy of Stored Ions. I. Storage. Adv. Atom. Mol. Phys. 1967, 3, 53-72. 